\title{
DIASPIDÍDEOS E PARASITOIDES ASSOCIADOS AO CULTIVO DA VIDEIRA NO RIO GRANDE DO SUL, BRASIL ${ }^{1}$
}

\author{
VERA REGINA DOS SANTOS WOLFF ${ }^{2}$, MARCOS BOTTON $^{3}$, DANIELE CAMPOS DA SILVA ${ }^{4}$
}

RESUMO - As cochonilhas-com-escudo (Hemiptera, Diaspididae) têm causado prejuízos significativos à cultura da videira no Rio Grande do Sul, principalmente em Vitis labrusca L. da cv. Niágara. No período de setembro de 2009 a outubro de 2011, foi realizado um inventário das espécies de Diaspididae ocorrentes no tronco das videiras e dos parasitoides associados. Foram avaliadas 32 propriedades localizadas nos municípios de Bento Gonçalves, Caxias do Sul, Flores da Cunha e Sarandi-RS. A espécie mais frequente e abundante foi Hemiberlesia lataniae (Signoret, 1869). Melanaspis arnaldoi (Costa Lima, 1924) e Pseudaonidia marquesi Costa Lima, 1924 foram registradas pela primeira vez em V. labrusca. Todas as espécies de cochonilhas apresentaram perfurações nos escudos indicando níveis de parasitismo natural que variaram de 11,3 a 76,5\% para H. lataniae, de 9 a 58,8\% para M. arnaldoi e de 3,4 a 22,2\% para P. marquesi. As seguintes associações entre parasitoides e cochonilhas foram encontradas: Encarsia sp. (Aphelinidae), Plagiomerus sp. (Encyrtidae), Amitus sp. (Platygastridae) e o hiperparasitoide Chartocerus sp. (Signiphoridae) a H. lataniae; Homalopoda sp. (Encyrtidae) a P. marquesi e Chartocerus sp. a M. arnaldoi. Amitus sp., Homalopoda sp. e Chartocerus $\mathrm{sp}$. foram associados pela primeira vez com estes diaspidídeos.

Termos para indexação: Hemiberlesia lataniae, Melanaspis arnaldoi, Pseudaonidia marquesi, Diaspididae, Vitis labrusca.

\section{DIASPIDIDAE AND ASSOCIATED PARASITOIDS IN "NIAGARA" CULTIVARS IN THE STATE OF RIO GRANDE DO SUL, BRAZIL}

\begin{abstract}
The Armored Scale insects (Hemiptera, Diaspididae) have caused damage to vineyards in Rio Grande do Sul, mainly Vitis labrusca L. cv. Niagara. From September 2009 to October 2011 we conducted a survey of Diaspididae species and associated parasitoids occurring in the trunk of the vines. It was evaluated 32 vineyards located in Bento Gonçalves, Caxias do Sul, Flores da Cunha and Sarandi, RS. The most frequent and abundant specie of armored scale insect was Hemiberlesia lataniae (Signoret, 1869). Melanaspis arnaldoi (Costa Lima, 1924) and Pseudaonidia marquesi (Costa Lima, 1924) were recorded for the first time in $V$. labrusca. All these species showed perforations in the scale cover indicating the presence of parasitism in the field. The level of parasitism ranged from 11.3 to $76.5 \%$ for H. lataniae, from 9 to $58.8 \%$ for $M$. arnaldoi and 3.4 to $22.2 \%$ for $P$. marquesi. The following associations between armored scale insects and parasitoids were found: Encarsia sp. (Aphelinidae), Plagiomerus sp. (Encyrtidae), Amitus sp. (Platygastridae) and a hyperparasitoid Chartocerus sp. (Signiphoridae) with H. lataniae; Homalopoda sp. (Encyrtidae) with P. marquesi and Chartocerus sp. with M. arnaldoi. Amitus sp., Homalopoda sp. and Chartocerus sp. are the first records association for these armored scale insects.

Index terms: Hemiberlesia lataniae, Melanaspis arnaldoi, Pseudaonidia marquesi, Diaspididae, Vitis labrusca.
\end{abstract}

${ }^{1}$ (Trabalho 145-13). Recebido em: 04-04-2013. Aceito para publicação em: 12-09-2014.

${ }^{2}$ Bióloga, D.Sc., Fepagro, Rua Gonçalves Dias 570, 90130-060 Porto Alegre, RS. Brasil, Pesquisadora visitante Embrapa Uva e Vinho. E-mail: vera-wolff@fepagro.rs.gov.br

${ }^{3}$ Eng. Agr., D.Sc., Embrapa Uva e Vinho. Rua Livramento 515, CP 130, 95700-000 Bento Gonçalves, RS. Brasil. E-mail: marcos. botton@embrapa.br

${ }^{4}$ Bióloga, M.Sc. Fepagro. E-mail: danientomo@yahoo.com.br. 


\section{INTRODUÇÃO}

A viticultura é uma atividade de grande importância econômica e social no Brasil, sendo o Rio Grande do Sul o maior produtor de uvas para processamento do País. A cultivar Niágara (Vitis labrusca) é uma das melhores alternativas de cultivo nas principais regiões produtoras, pois é uma uva de fácil manejo, de grande aceitação no mercado além de poder ser consumida in natura e processada para elaboração de vinho comum (CAMARGO et al., 2011).

Dentre as pragas mais comuns que atacam a Niágara, destacam-se as cochonilhas-com-escudo ou carapaça (Hemiptera, Diaspididae), as quais podem provocar a morte das plantas (BOTTON et al., 2003). Estas cochonilhas ocorrem principalmente no tronco das plantas, sendo semelhantes quanto ao tamanho e forma, dificultando a identificação no campo. Praticamente não existem informações sobre a bioecologia destas cochonilhas na cultura da videira, o que dificulta o estabelecimento de medidas de controle no período de eclosão das ninfas, fase em que o inseto é mais sensível (BOTTON et al., 2012).

Até o momento, não foi realizado um inventário das espécies que ocorrem em Niágara, bem como dos parasitoides a elas associados, os quais podem auxiliar num programa sustentável de manejo.

No mundo, são conhecidas 61 espécies de diaspidídeos em Vitis spp., das quais 23 ocorrem no Brasil (CLAPS et al., 1999, 2001; BEN-DOV et al. 2014). Hemiberlesia lataniae Signoret e Duplaspidiotus fossor Newstead citadas como pragas de Niágara (BOTTON et al., 2003) . Segundo Botton e colaboradores (2012), quando ocorrem infestações elevadas destas cochonilhas, o controle químico é recomendado, porém alertam para as dificuldades do contato do produto com as cochonilhas, protegidas pela localização abaixo do ritidoma. Além disso, observam que as aplicações devem ser realizadas somente nos focos de infestação para preservar os parasitoides que realizam o controle biológico natural das cochonilhas. Estas informações são mencionadas de forma prática, dado o grande número de escudos perfurados observados nas infestações no campo sem o conhecimento das espécies presentes e da melhor época de aplicação.

Este trabalho foi realizado com o objetivo de conhecer as espécies de Diaspididae presentes no cultivo de V. labrusca cv. Niágara nas principais regiões produtoras do Rio Grande do Sul, bem como os parasitoides associados e o nível de parasitismo natural nos vinhedos.

\section{MATERIAL E MÉTODOS}

O estudo foi desenvolvido no período de setembro de 2009 a fevereiro de 2010 (primeira etapa) e de junho de 2010 a outubro de 2011 (segunda etapa).

Na primeira etapa, foram selecionadas trinta propriedades com cultivo de Niágara, indicadas por agentes da extensão rural, em municípios considerados polos produtores desta cultivar no Estado do Rio Grande do Sul. Foram amostrados 10 vinhedos em Bento Gonçalves (14 e 15-09-2009), 10 em Caxias do Sul ( $1^{\circ}$ e 02-12-2009), cinco em Flores da Cunha (03-12-2009) e cinco em Sarandi (09-02-2010), todos com histórico de infestação de cochonilhas no tronco.

Em cada propriedade, foram recolhidas amostras de cochonilhas em 10 plantas infestadas, retirando-se com um estilete cerca de $2 \mathrm{~cm}$ x $10 \mathrm{~cm}$ da casca do caule. Cada amostra foi individualizada em potes de plástico com tampa, etiquetada e transportada ao Laboratório de Entomologia da Fundação Estadual de Pesquisa Agropecuária FEPAGRO, Sede, em Porto Alegre, para serem triadas. As cochonilhas foram examinadas sob microscópio estereoscópico, sendo quantificadas quanto à presença ou não de perfurações nos escudos, devido ao ataque de parasitoides.

Embora o inventário tenha sido realizado em datas e locais diferentes, foi possível observar níveis de parasitismo através da proporção entre o número de escudos íntegros e de perfurados, indicando a incidência de parasitoides em fêmeas adultas dos diaspidídeos.

Para a identificação das cochonilhas, foram separadas fêmeas adultas, as quais foram preparadas em lâminas microscópicas temporárias e permanentes. Para tal, utilizou-se do método adaptado de Claps e DeHaro (1995), as cochonilhas foram colocadas em uma placa escavada de porcelana, imersas em solução de $\mathrm{KOH}$ a $10 \%$, aquecidas por 30 minutos em forno a $40^{\circ} \mathrm{C}$. A seguir, foram lavadas com água destilada apertando-se com uma agulha histológica até ser extraído seu conteúdo. Logo foram transportadas para uma solução de Essig e Fucsina ácida para corar, desidratadas em sequência com álcool a $70 \%$ e $96 \%$ e por último colocadas em essência de óleo de cravo, cerca de 2 min em cada solução. As lâminas permanentes foram montadas com bálsamo do Canadá.

As lâminas foram examinadas ao microscópio óptico utilizando-se, para a identificação das espécies, da bibliografia e da comparação com exemplares da coleção do Museu de Entomologia Ramiro Gomes 
Costa (MRGC), da Fundação Estadual de Pesquisa Agropecuária (FEPAGRO). Exemplares de cada espécie das cochonilhas coletadas foram depositados na coleção entomológica do MRGC.

$\mathrm{Na}$ segunda etapa, foram selecionadas 3 propriedades (FEPAGRO - Serra do Nordeste, Novello e Rossi) localizadas no município de Caxias do Sul, com infestação de cochonilhas no tronco. Na FEPAGRO Serra do Nordeste, o plantio das videiras, todas da varieda de Niágara, foi realizado em 1981, utilizando manejo convencional e realizando tratamentos com Curzate, Sulfato de Cobre, Manzate, Cercobin e Klap. Na Novello, o plantio ocorreu em 1982, e as variedades Isabel, Niágara e Seyve Villard estão misturadase foi utilizado o manejo convencional, aplicando Actara, Dithane e cobre. Na Rossi, o plantio foi em 1980, variedade foi Niágara, sendo realizado o manejo orgânico, utilizando principalmente Ecolife.

Os procedimentos para a obtenção das amostras, triagem, preparo e identificações das cochonilhas foram os mesmos utilizados na primeira etapa. No entanto, nesta fase, as amostragens foram realizadas ao longo do ano, englobando a primavera (05-10-2010, 03-11-2010, 1'-12-2010), verão (10-012011, 14-02-2011, 17-03-2011), outono (19-04-2011, $1^{\circ}$-06-2011) e inverno (05-07-2011, 13-09-2011).

Em cada propriedade, foram marcadas 15 plantas, que foram amostradas para identificação das espécies presentes. Ao longo das avaliações, foi registrada a fase de desenvolvimento (ovo, ninfa de primeiro instar, fêmeas adultas) e anotados o número de escudos íntegros e os com perfurações, que indicam a emergência de parasitoides.

Duas outras plantas foram amostradas em cada propriedade, em cada avaliação, para a obtenção dos parasitoides. As cochonilhas sem perfuração no escudo foram identificadas, isoladas e colocadas em cápsulas de gelatina, as quais foram etiquetadas e fixadas com alfinetes em bandejas de isopor e deixadas na temperatura ambiente do laboratório. As cápsulas foram examinadas diariamente para verificar a emergência dos parasitoides que, após a morte, foram conservados em cápsulas com algodão, dentro de caixas com naftalina ou em tubos com álcool a 70\%, para posterior identificação. Os parasitoides foram enviados para o Departamento de Entomologia do Natural History Museum (NHM), em Londres, onde foram preparados e identificados.

\section{RESULTADOS E DISCUSSÃO}

Hemiberlesia lataniae foi encontrada em todas as amostras de V. labrusca das variedades Niágara, coletadas no Rio Grande do Sul. Esta espécie de Diaspididae possui ampla distribuição geográfica, sendo encontrada em 480 hospedeiros, dos quais cerca de 40 ocorrem no Brasil (CLAPS et al., 2001; BEN-DOV et al., 2013).

O escudo ou carapaça que reveste o corpo da fêmea adulta desta espécie é arredondado, achatado, medindo até $2 \mathrm{~mm}$ de diâmetro, com coloração que varia do branco acinzentado ao marrom-claro, com exúvias subcentrais amarelo-claras, podendo ser confundido com a coloração da casca da videira. Embaixo do escudo, encontra-se o corpo da fêmea adulta, que é oval, membranoso, medindo cerca de $1 \mathrm{~mm}$. No pigídio há quatro grupos de glândulas circungenitais, o $1^{\circ}$ par de lóbulos é largo e proeminente e o ânus é grande, próximo da margem pigidial (CLAPS; WOLFF, 2003).

Em relação à fenologia do ataque, durante todo o período de avaliação, foram encontradas cochonilhas vivas, exceto nos meses de janeiro, agosto e outubro de 2011, indicando que a espécie permanece alimentando-se durante praticamente todo o ano. Provavelmente, a espécie completa três a quatro gerações durante o ano, indicadas pela presença de fêmeas com ovos nos meses de outubro e novembro de 2010, abril, junho e setembro de 2011, e de ninfas de primeiro instar nos meses de setembro de 2010 , fevereiro e abril de 2011. A realização de tratamentos visando ao controle de ninfas de primeiro instar, fase em que o inseto é mais suscetível, deveria ser feita nos meses de fevereiro, abril e setembro (verão, início do outono e final de inverno) para $H$. lataniae. Porém, medidas para preservar o controle biológico natural fazem-se necessárias, uma vez que os parasitoides estão presentes praticamente durante todo o ano.

$\mathrm{Na}$ primeira etapa, dependendo do parreiral avaliado, o percentual variou de 11,3 a $57,5 \%$ de parasitismo. Este fato indicou haver um nível significativo de parasitoides que ocorrem naturalmente nos vinhedos (Tabela 1).

$\mathrm{Na}$ segunda etapa, a variação a campo de parasitismo na FEPAGRO - Serra do Nordeste, foi de 29,4 a 58,5\%; na propriedade Rossi, foi de 26,4 a $46,8 \%$ e, na propriedade Novello, entre 11,7 e $76,5 \%$ (Tabela 2). Esta discrepância na propriedade Novello pode estar relacionada com a ocorrência de H. lataniae em apenas cinco plantas amostradas, diferente das outras propriedades, onde a mesma ocorreu em todas as amostras. 
Os parasitoides que emergiram de $H$. lataniae, provenientes das coletas realizadas em Caxias do Sul, foram: três exemplares de Encarsia sp. (Aphelinidae), dois Plagiomerus sp. (Encyrtidae), um Amitus sp. (Platygastridae) e nove do hiperparasitoide Chartocerus sp. (Signiphoridae); da propriedade Rossi, foram obtidos Encarsia sp. (1), Amitus sp. (1) e Chartocerus sp. (2); da Fepagro, um Plagiomerus sp.; os demais parasitoides emergiram das coletas realizadas nas propriedades da primeira etapa.

Já foram observadas Encarsia aurantii (Howard), E. citrina (Crawford), E. inquirenda (Silvestri, 1930) e E. lounsburyi (Berlese e Paoli), Plagiomerus bangaloriensis Shafee, Alam \& Agarwal e $P$. diaspidis Crawford associadas a $H$. lataniae (MOUSTAFA; ABD-RABOU, 2011; BENDOV et al., 2013;NATURAL HISTORY MUSEUM, 2014).

Foram encontradas, além de $H$. lataniae, duas outras espécies de diaspidídeos, localizadas principalmente na axila dos ramos das videiras. Os escudos das fêmeas adultas são muito semelhantes entre si, bastante duros, subcirculares, convexos, com cerca de $1 \mathrm{~mm}$ de diâmetro, coloração escura, quase preta, parte ventral da mesma cor da dorsal, plana, aderente à casca da planta, internamente revestidos por cera esbranquiçada. Porém, estas cochonilhas diferem na forma do corpo e principalmente nos caracteres encontrados no pigídio.

Na FEPAGRO - Serra do Nordeste, foi identificada uma fêmea adulta viva de Melanaspis arnaldoi (Costa Lima, 1924) em setembro de 2010, e apenas escudos secos desta cochonilha, em duas plantas de Niágara, nos meses de dezembro e fevereiro de 2010 e abril, junho, julho e setembro de 2011.

O corpo da fêmea adulta de $M$. arnaldoi é subcircular, com uma reentrância lateral, na altura do pigídio. No pigídio, não há glândulas circungenitais; há quatro pares de lóbulos desenvolvidos, com as margens arredondadas, sendo que o $4^{\circ}$ lóbulo é um pouco menor; há um par de paráfises curtas no prolongamento do lado interno do $1^{\circ}$ par de lóbulos; dois pares de paráfises longas, um que se origina na incisura entre o $1^{\circ}$ e o $2^{\circ}$ pares de lóbulos e o outro par de paráfises, maiores e mais dilatadas, na incisura entre o $2^{\circ}$ e $3^{\circ}$ pares de lóbulos; após o $4^{\circ}$ lóbulo, a margem do pigídio apresenta-se espessada por pequenas paráfises (COSTA LIMA, 1924).

Foi possível observar, em condições de campo, perfurações nos escudos de $M$. arnaldoi que variaram de 9 a 58,8 \%, e, em laboratório, emergiram dois exemplares de Chartocerus sp. (Tab. 3).

$\mathrm{Na}$ propriedade Novello, onde havia as variedades Niágara (5), Isabel (9) e Seyve Villard (3), foram encontrados escudos e fêmeas adultas vivas de Pseudaonidia marquesi Costa Lima, 1924 durante todo o período de avaliação, exceto fêmeas vivas nos meses de março, agosto e setembro de 2011; e de setembro a fevereiro de 2011, foram encontradas ninfas de primeiro instar. Para esta espécie, de setembro a março (final de inverno, primavera e verão), são os meses mais adequados para a realização de tratamentos visando ao controle de ninfas de primeiro instar.

O corpo da fêmea adulta de $P$. marquesi é subcircular, com uma constrição nas laterais da região cefalotorácica e pequeno grupo de poros glandulares acima dos espiráculos anteriores. A parte central do pigídio apresenta uma área reticulada e sem glândulas circungenitais. Na margem pigidial, há dois pares de lóbulos desenvolvidos, arredondados, e os demais pouco se distinguem; quatro pares de paráfises, uma em cada incisão entre os lóbulos pigidiais, sendo as maiores as que se encontram entre o $1^{\circ}$ e $2^{\circ}$ pares de lóbulos, e as demais vão decrescendo, e possuem um par de placas na incisura mediana (COSTA LIMA, 1924).

Foi possível observar níveis de parasitismo natural que variaram entre 3,4 e $22,2 \%$ em $P$. marquesi, e da coleta de março de 2011 foi obtido um parasitoide (Homalopoda sp. - Encyrtidae) (Tab. 4). 
TABELA 1-Número de escudos de fêmeas de Hemiberlesia lataniae, não perfurados (NP) e perfurados (P) pela emergência de parasitoides a campo e percentagem de parasitismo $(\% \mathrm{P})$, nas datas de avaliação.

\begin{tabular}{|c|c|c|c|c|c|c|c|c|c|c|c|c|}
\hline \multirow{2}{*}{$\begin{array}{l}\text { Vinhedo } \\
\text { Planta } \\
\end{array}$} & \multicolumn{3}{|c|}{$\begin{array}{l}14 \text { e } 15-09-2009 \\
\text { Bento Gonçalves }\end{array}$} & \multicolumn{3}{|c|}{$\begin{array}{c}1^{\circ} \text { e } 02-12-2009 \\
\text { Caxias do Sul }\end{array}$} & \multicolumn{3}{|c|}{$\begin{array}{c}\text { 03-12-2009 } \\
\text { Flores da Cunha }\end{array}$} & \multicolumn{3}{|c|}{$\begin{array}{l}\text { 09-02-2010 } \\
\text { Sarandi }\end{array}$} \\
\hline & NP & $\mathrm{P}$ & $\% \mathrm{P}$ & NP & $\mathrm{P}$ & $\% \mathrm{P}$ & NP & $\mathrm{P}$ & $\% \mathrm{P}$ & NP & $\mathrm{P}$ & $\% \mathrm{P}$ \\
\hline 1 & 1055 & 302 & 22,2 & 819 & 238 & 22,5 & 417 & 103 & 19,8 & 261 & 169 & 39,3 \\
\hline 2 & 1296 & 520 & 28,6 & 85 & 30 & 26,0 & 420 & 151 & 26,4 & 133 & 180 & 57,5 \\
\hline 3 & 1436 & 948 & 39,7 & 6885 & 1308 & 15,9 & 515 & 315 & 37,9 & 426 & 196 & 31,5 \\
\hline 4 & 361 & 206 & 36,3 & 51 & 7 & 12,0 & 431 & 212 & 32,9 & 397 & 300 & 43,0 \\
\hline 5 & 48 & 13 & 21,3 & 2754 & 390 & 12,4 & 285 & 169 & 37,2 & 457 & 171 & 27,2 \\
\hline 6 & 4086 & 851 & 17,2 & 180 & 110 & 37,9 & - & - & - & - & - & - \\
\hline 7 & 1239 & 607 & 32,8 & 0 & 0 & 0 & - & - & - & - & - & - \\
\hline 8 & 452 & 151 & 25,0 & 747 & 144 & 16,1 & - & - & - & - & - & - \\
\hline 9 & 78 & 10 & 11,3 & 368 & 127 & 25,6 & - & - & - & - & - & - \\
\hline 10 & 446 & 169 & 27,4 & 433 & 137 & 24,0 & & & & & & \\
\hline Médias $\pm E P$ & & $1 \pm$ & & & $9,2 \pm$ & & & $0,8 \pm$ & & & $9,7 \pm$ & \\
\hline
\end{tabular}

TABELA 2-Número de escudos de fêmeas de Hemiberlesia lataniae, não perfurados (NP) e perfurados (P) pela emergência de parasitoides a campo, e percentagem de parasitismo (\% P), nas datas de avaliação, em Caxias do Sul.

\begin{tabular}{cccccccccc}
\hline $2^{\text {a }}$ etapa & \multicolumn{3}{c}{ FEPAGRO Serra do Nordeste } & \multicolumn{3}{c}{ Propriedade Rossi } & \multicolumn{3}{c}{ Propriedade Novello } \\
\hline Coletas & NP & P & $\%$ P & NP & P & $\%$ P & NP & P & $\%$ P \\
\hline Out/2010 & 741 & 374 & 33,5 & 1609 & 748 & 31,7 & 0 & 0 & 0 \\
Nov/2010 & 421 & 371 & 46,8 & 895 & 528 & 37,1 & 0 & 0 & 0 \\
Dez/2010 & 557 & 259 & 31,7 & 591 & 455 & 43,4 & 17 & 7 & 29,1 \\
Jan/2011 & 624 & 351 & 36,0 & 880 & 316 & 26,4 & 11 & 36 & 76,5 \\
Fev/2011 & 283 & 118 & 29,4 & 791 & 336 & 29,8 & 15 & 2 & 11,7 \\
Mar/2011 & 119 & 111 & 48,2 & 361 & 318 & 46,8 & 0 & 0 & 0 \\
Abr/2011 & 337 & 148 & 30,5 & 507 & 243 & 32,4 & 28 & 10 & 26,3 \\
Jun/2011 & 138 & 195 & 58,5 & 423 & 186 & 30,5 & 0 & 0 & 0 \\
Jul/2011 & 178 & 93 & 34,3 & 357 & 282 & 44,1 & 2 & 0 & 0 \\
Set/2011 & 149 & 89 & 37,3 & 335 & 253 & 43,0 & 22 & 3 & 12,0 \\
\hline Médias \pm EP & \multicolumn{1}{c}{$38,6 \pm 2,9$} & \multicolumn{4}{c}{$36,5 \pm 2,3$} & & $15,5 \pm 7,6$ & \\
\hline
\end{tabular}

TABELA 3- Número de escudos de fêmeas de Melanaspis arnaldoi, não perfurados (NP) e perfurados (P) pela emergência de parasitoides a campo, e percentagem de parasitismo (\% P), nas datas de avaliação.

\begin{tabular}{cccc}
\hline $2^{\mathrm{a}}$ etapa & \multicolumn{3}{c}{ FEPAGRO Serra do Nordeste } \\
\hline Coletas & NP & $\mathrm{P}$ & $\% \mathrm{P}$ \\
\hline Out $/ 2010$ & 0 & 0 & 0 \\
Nov/2010 & 0 & 0 & 0 \\
Dez/2010 & 7 & 10 & 58,8 \\
$\mathrm{Jan} / 2011$ & 0 & 0 & 0 \\
$\mathrm{Fev} / 2011$ & 20 & 2 & 9,0 \\
$\mathrm{Mar} / 2011$ & 0 & 0 & 0 \\
$\mathrm{Abr} / 2011$ & 14 & 7 & 33,3 \\
$\mathrm{Jun} / 2011$ & 9 & 5 & 35,7 \\
$\mathrm{Jul} / 2011$ & 10 & 0 & 0 \\
$\mathrm{Set} / 2011$ & 7 & 9 & 56,2 \\
\hline Média $\pm \mathrm{EP}$ & \multicolumn{4}{c}{$19,3 \pm 7,71$} \\
\hline
\end{tabular}


TABELA 4 - Número de escudos de fêmeas de Pseudaonidia marquesi, não perfurados (NP) e perfurados (P) pela emergência de parasitoides a campo, e percentagem de parasitismo (\% P), nas datas de avaliação.

\begin{tabular}{cccc}
\hline $2^{\mathrm{a}}$ etapa & \multicolumn{3}{c}{ Propriedade Novello } \\
\hline Coletas & $\mathrm{NP}$ & $\mathrm{P}$ & $\% \mathrm{P}$ \\
Out $/ 2010$ & 465 & 69 & 12,9 \\
Nov/2010 & 472 & 17 & 3,4 \\
Dez/2010 & 442 & 26 & 5,5 \\
$\mathrm{Jan} / 2011$ & 471 & 66 & 12,2 \\
$\mathrm{Fev} / 2011$ & 142 & 25 & 14,9 \\
$\mathrm{Mar} / 2011$ & 192 & 55 & 22,2 \\
$\mathrm{Abr} / 2011$ & 410 & 28 & 6,3 \\
$\mathrm{Jun} / 2011$ & 189 & 39 & 17,1 \\
$\mathrm{Jul} / 2011$ & 140 & 8 & 5,4 \\
$\mathrm{Set} / 2011$ & 265 & 24 & 8,3 \\
\hline Média $\pm \mathrm{EP}$ & \multicolumn{4}{c}{$10,8 \pm 1,91$} \\
\hline
\end{tabular}

\section{CONCLUSÕES}

A principal espécie de Diaspididae que ocorre associada ao tronco na cultivar Niágara Rosada e Branca, no Rio Grande do Sul, é Hemiberlesia lataniae. Melanaspis arnaldoi e Pseudaonidia marquesi são registradas pela primeira vez na cultivar.

Este é o primeiro registro da associação de H. lataniae com Amitus sp. e Chartocerus sp.; de M. arnaldoi com Chartocerus sp. e de P. marquesi com Homalopoda sp.

O parasitismo natural varia de 11,3 a $76,5 \%$ para H. lataniae, de 9 a 58,8\% para $M$. arnaldoi e de 3,4 a $22,2 \%$ para $P$. marquesi.

O controle das ninfas de primeiro instar de $H$. lataniae, no Rio Grande do Sul, deve ser realizado nos meses de fevereiro, abril e setembro (verão, início do outono e final de inverno).

\section{AGRADECIMENTOS}

Ao Dr. John Noyes, do Natural History Museum ,pela identificação dos parasitoides; às biólogas Cristine Elise Pulz, Juliana Conte Zanotelli e ao Eng. Agr. Nelson Bertoldo, pelo auxílio nas coletas. Ao CNPq, pela Bolsa de Pesquisador Visitante. À FEPAGRO, pelos veículos e combustíveis utilizados para as coletas nos municípios do RS. À Dra. Caren Regina Cavichioli Lamb, da FEPAGRO - Serra do Nordeste. À família Rossi, na linha 60, e à família Novello, na Fazenda Souza, de Caxias do Sul, por permitirem a realização da pesquisa nestas propriedades, Aos demais produtores visitados na primeira etapa e aos técnicos da EMATER, que auxiliaram nos contatos com estes produtores.

\section{REFERÊNCIAS}

BEN-DOV, Y.; MILLER, D. R.; GIBSON, G. A. P. ScaleNet. $<$ http://www.sel.barc.usda.gov/scalecgi/ scaleson.e?family $=\&$ scalefamily $=$ Diaspididae $\& g$ enus $=$ Vitis\&scalegenus $=\&$ species $=>$. Acesso em: 24 nov. 2014.

BOTTON, M. ; MAIA, J. D. G. ; SPECHT, A. ; MACHOTA Jr, R. ; ZENKER, M. M . Pragas da uva Niágara no Brasil. In: JOÃO DIMAS GARCIA MAIA; UMBERTO ALMEIDA CAMARGO. (Org.). O cultivo da videira Niágara no Brasil. Brasília: Embrapa Informação Tecnológica, 2012. v. 1, p. 229-251.

CAMARGO, U. A.; TONIETTO, J.; HOFMANN, A. Progressos na viticultura brasileira. Revista Brasileira de Fruticultura, Jaboticabal, v. 33, p.144-149, 2011. Número Especial.

Catálogo de las Diaspididae (Hemiptera: Coccoidea) exóticas de la Argentina, Brasil y Chile. Revista de La Sociedad Entomológica Argentina, San Miguel de Tucumán, v.3, p.1-59, 2001.

CLAPS, L. E.; De HARO, M. E. Conociendo nuestra fauna IV. Familia Diaspididae (Insecta: Homoptera). Morfologia y biologia. Serie Monografica y Didactica Facultad de Ciencias Naturales e Instituto Miguel Lillo UNT, Tucuman, v.20, p.1-21, 1995. 
CLAPS, L. E.; WOLFF, V. R. S. Cochinillas Diaspididae (Hemiptera: Coccoidea) frecuentes en plantas de importancia económica de la Argentina y Brasil. Revista de La Sociedad Entomológica Argentina, Buenos Aires, n.3, p.1-59, 2003.

CLAPS, L. E.; WOLFF, V. R. S.; GONZÁLEZ, R. H. Catálogo de las especies de Diaspididae (Hemiptera, Coccoidea) nativas de Argentina, Brasil y Chile. Insecta Mundi, Gainsville, v.13, n.3/4, p. 239-256, 1999.

CLAPS, L. E.; WOLFF, V. R. S.; GONZÁLEZ, R. H. Catálogo de las espécies de Diaspididae (Hemiptera, Coccoidea) exóticas de Argentina, Brasil y Chile. Revista de La Sociedad Entomológica Argentina, Buenos Aires, v. 60, n.1-4, p. 9-34, 2001.
COSTA LIMA, A. Sobre insectos parasitas da videira. Almanak Agricola Brasileiro, Rio de Janeiro, p.135-141, 1924. MOUSTAFA, M.; ABD-RABOU, S. Natural enemies of the lataniae scale, Hemiberlesia lataniae (Hemiptera: Diaspididae) in Egypt. Egyptian Academic Journal of Biological Sciences, Abbasia, v. 4, n. 1, p. 75-90, 2011.

NATURAL HISTORY MUSEUM. Universal Chalcidoidea Database. Disponivel em: $<$ http:// www.nhm.ac.uk/research-curation/research/ projects/chalcidoids/database/namedHost. dsml?HOSGENUS=Hemiberlesia $>$ Acesso em: 7 mar.2014. 